\title{
Outcomes of lenalidomide therapy in angioimmunoblastic T-cell lymphoma.
}

Gorenkova L. G., Kravchenko S. K., Kovrigina A. M., Klebanova E. E.

National Research Centre of Haematology. Russian Federation, Moscow

Angioimmunoblastic T-cell lymphoma accounts for $15 \%$ - 20 $\%$ of all nodal T-cell lymphomas and is characterized by a range of specific features distinguishing it from other forms of T-cell lymphoproliferative disorders: an abundant proliferating network of vessels surrounding the tumour cells, EBV-infected B cells in the microenvironment, combination with autoimmune disorders, and hypergammaglobulinaemia. No effective, optimal induction therapy has been established to date; overall survival does not exceed $30 \%$ in patients receiving short-pulse therapy with anthracycline antibiotics, and not more than $20 \%$ of patients with relapsing \refractory disease achieve a second complete remission.
Another problem in the treatment of AITL is choosing a therapy for older patients (over 65 years old) and / or patients with high comorbidity index values. Lenalidomide is a drug exhibiting immunomodulatory and antiangiogenic activity. A small number of studies have been conducted since 2010 on the use of this drug in the treatment of refractory T-cell lymphomas, which demonstrated its potential efficacy, particularly in patients with angioimmunoblastic T-cell lymphoma.
The goal: to evaluate the efficacy of lenalidomide in different regimens administered to patients with AITL: first-line therapy in elderly patients, treatment of progressive disease, maintenance therapy.

The study included 20 patients with a confirmed diagnosis of AITL.

Results: 8 patients received lenalidomide maintenance therapy after completion of the ALL-2009 protocol, 7 of them are now in a complete remission; 8 received lenalidomide as salvage therapy for progressive disease: 5 of them achieved a CR/PR, while the disease was still progressive in the other 3 . The most interesting subset was Group 3 in which lenalidomide was administered as first-line therapy (median age 68 years) and 3 out of 4 subjects achieved a complete remission of the disease while 1 patient died of unrelated causes at the start of treatment.

Therefore, the reported study conducted in a small patient group indicated that lenalidomide is a potentially effective agent for the treatment of AITL both as maintenance therapy and as a choice for refractory disease. The drug was also able

to induce an antitumour response when given as first-line therapy in elderly patients with somatic diseases. 